\title{
On the Performance of Inertial/GNSS/Doppler Velocity Log Integrated Navigation Systems for Marine Applications
}

\author{
M. Romanovas $^{1}$, L. Lança ${ }^{1}$, R. Ziebold $^{1}$ \\ ${ }^{1}$ Institute of Communication and Navigation \\ German Aerospace Centre (DLR) \\ Kalkhorstweg 53 \\ 17235 Neustrelitz \\ GERMANY
}

Inertial Sensors and Systems 2015

Karlsruhe, Germany 


\begin{abstract}
Although the GNSS/GPS had become the primary source for Positioning, Navigation and Timing (PNT) information in maritime applications, the ultimate performance of the system can strongly degrade due to space weather events, deliberate interference, shadowing, multipath and overall system failures. Within the presented work the development of an affordable integrated PNT unit for future on-board integrated systems is presented, where the GNSS information is fused both with inertial and Doppler Velocity Log (DVL) measurements. Here redundant and complementary information from different sensors serves to improve the system performance and reduce the position drift when the GNSS signals are not available. The nonlinearity of this advanced fusion problem is addressed by employing different forms of Sigma-Points Kalman Filter (SPKF) and further detailed analysis is presented in terms of the process and measurement models implemented. The results demonstrate that position drift can be significantly reduced by incorporating DVL measurements in IMU/GNSS system and that the proposed integrated navigation algorithm is feasible and efficient for GNSS outages of prolonged duration, where pure inertial GNSS outage bridging would be either inaccurate or would require too expensive IMUs.
\end{abstract}

\title{
1. Introduction
}

Nowadays the process of vessel navigation is supported by a variety of independent sources of navigational information. The Global Navigation Satellite Systems (GNSS), in particular the Global Positioning System (GPS) is considered to be the key component in maritime navigation for provision of an absolute position, velocity and precise time (PVT) information. However, the GNSS receiver is usually not fully integrated with other already existing on-board sensors (e.g. Velocity Doppler Log (DVL), gyrocompass, etc.). Navigators are responsible of choosing a system/sensor type, system settings and interpretation of each subsystem output as well as for monitoring the actual response of the vessel. In spite of all the efforts, $50 \%$ of all accidents in the Baltic Sea during 2011 were caused by navigational errors including human factors, misinterpretation of navigational data or incorrect decision making [1]. In order to support the decision making and improve the safety of berth-to-berth navigation process, the International Maritime Organization (IMO) had started the e-Navigation initiative, where a resilient provision of Positioning, Navigation and Timing (PNT) data is considered as to be the key enabler.

The recognized vulnerability of GNSS in certain environments introduces concerns to the provision of on-board reliable navigational data required in maritime safety-critical 
operations. The IMO e-navigation strategic implementation plan aims to improve the reliability and resilience of on-board PNT information through both the enhancement of existing sensors and the augmentation with external information sources. The presented work addresses the limitations of GNSS-only systems by its integration with other onboard navigation sensors like DVL and inertial sensors (Inertial Measurement Unit - IMU) within a special data processing unit [2]. Here the integration of multiple sensors with independent error patterns highly improves the overall system resilience against GNSS channel contamination and is crucial for providing high integrity PNT data.

Although the benefits of integrated IMU/GNSS navigation system have been already demonstrated for marine applications [3], the scenarios with GNSS signal outages up to 510 minutes still put too demanding requirements on the performance of inertial sensors. The presented paper demonstrates how the PNT performance during signal outages can be improved by augmenting the IMU/GNSS system with a DVL using Sigma Points Kalman Filtering (SPKF) framework with attitude quaternion parametrization [4]. Here we still follow a classical design approach where the inertial measurements are considered as a core sensing modality and provides a complete navigation solution (position, velocity and attitude), while both GNSS and DVL measurements are used as complementary aiding measurements in order to reduce the fast drift of a pure inertial solution. Clearly, adoption of the direct strapdown inertial mechanization allows us to avoid any explicit assumptions regarding the underlying models and even subtle vessel motions can be tracked using this classical approach. The work demonstrates that although a classical IMU/GNSS integration approach is able to provide horizontal position accuracy up to 10 meters for GNSS signal outages shorter than few minutes, the incorporation of DVL 2D velocity information extends the period of standalone navigation within accuracy requirements for longer than 5 minutes. Moreover, the performance becomes far less sensitive to IMU quality and lower cost inertial sensors such as Micro Electro Mechanical Systems (MEMS)-based ones can be adopted. This complements our previous findings in [5] and, although the main characteristics of MEMS sensors are still inferior of those of more expensive FOG-based systems, their accuracy is sufficient for certain application scenarios such as coasting GNSS outages of shorter duration, supporting Fault Detection and Exclusion (FDE) functionality as well as smoothing of GNSS navigation solutions. The main objective of this work is to demonstrate the feasibility of the integrated IMU/GNSS/DVL navigation solution using real measurement campaigns and to provide a 
systematic analysis on the performance of proposed hybrid navigation solution including an analysis on chosen SPKF scheme, filter design and measurement model selection/

The rest of the paper is organized as follows. In Section 2 we provide a brief overview of the related work. Section 3 describes the relevant mathematical methods including the details on filter implementation and associated dynamical models. The section 4 introduces the measurement setup with the results shown in Section 5. Finally, Section 6 provides a concise discussion with the summary and outlook for future work given in Section 7.

\section{Related Work}

Among clear advantages of the inertial sensors one could mention that they are completely self-contained, immune to interference, highly dynamical, small size and often lightweight (especially MEMS-based). Unfortunately, the sensors provide only incremental information and the integration output drifts over time when no external reference is provided. However, inertial sensors have complementary properties to those of GNSS and both sensors are often integrated to improve navigation robustness resulting both in highly dynamical and drift-free system. IMU utilization allows to bridge short-term GNSS outages caused by signal blockage or antenna shadowing and even to support navigation in jammed environments if deep integration of GNSS raw data and inertial outputs is used. Finally, the accuracy of the combined system usually exceeds the specified accuracy of the GNSS alone and allows less than four satellites to play a role in the final navigation solution (in tightly-coupled architectures).

Augmentation of GNSS with inertial sensors in order to mitigate intentional or unintentional GNSS signal interference has a fairly long history $[6,7]$. Such systems are able to deliver position and velocity information at rapid update rate while preserving a low noise content due to the smoothing behavior of inertial integration. Since recently it has been also accepted that at least for classical IMU/GNSS integration there is almost no difference between classical error-state Extended KF and full-state UKF except of situations with unrealistically large initial uncertainties or scenarios with extremely high dynamics [8]. Important is that although IMU/GNSS fusion is a well-established technique for numerous applications, the IMU is not contained in the list of mandatory on-board navigation equipment and their wider acceptance is strongly conditioned on the price. Obviously, high performance IMUs are still prohibitively expensive with the price often above 30 kEuro and the inertial MEMS sensors due their continuous improvement in performance, provide a 
promising alternative especially when one considers the trade-off between bias in-run stability and the price. Increasingly, commercial systems [9] are becoming available which provide an integration of GNSS and MEMS IMUs.

The navigation systems for maritime applications have also relatively long history of integration using Extended KF (EKF) such as system mentioned in [10], where early GPS, speed log and Loran-C have been combined. The seminal work [3] also tried to assess the possibility to replace the FOG IMU with lower cost MEMS IMU in hybrid navigation systems and assessed the performance of the system under presence of GNSS faults in maritime scenarios. In our recent work [5] we have evaluated the impact of inertial sensor quality on the performance of hybrid IMU/GNSS system in maritime applications. The obtained results confirmed that the quality of the inertial sensor mainly affects the GNSS outage bridging (both position and heading), while the performance of FDE functionality as well as the accuracy (smoothing of GNSS noise) remained almost not affected by the quality of IMU.

A number of interesting works on IMU and DVL fusion can be found in the literature on Autonomous Underwater Vehicles (AUV) $[11,12,13]$ as they are required to navigate over extended periods of time at the absence of absolute reference information and usually employing only IMU-aided velocity measurements (typically those provided by DVL). The systems were reported to deliver relatively low navigation errors with the main error contribution due to scaling factors of the DVL and heading errors from the gyroscope [14]. Note that differently from AUV survey applications we cannot perform the navigation of the vessel in the confined area in commercial applications, and, therefore, the most the errors such as those due to heading and DVL scaling factor cannot simply cancel out in our scenario as it happens for AUVs when proper exploration path planning is used.

\section{Methods}

The methods of Recursive Bayesian Estimation (RBE) deal with the problem of estimating the changing in time state of system using only noisy observations and some a priori information regarding the underlying system dynamics. There are numerous advantages of the probabilistic paradigm where the most important are the ability to accommodate inaccurate models as well as imperfect sensors, robustness in real-world applications and often being the best known approach to many challenging navigation problems [15]. 
The RBE algorithms are used to estimate the state $x_{k}$ of a system at the time $t_{k}$ based on all measurements $Z_{k}=\left\{z_{0}, \ldots, z_{k}\right\}$ up to that time. Then any recursive Bayesian estimation cycle is performed in two steps:

Prediction: The a priori probability is calculated from the last a posteriori probability using the process model.

Correction: The a posteriori probability is calculated from the a priori probability using the measurement model and the current measurements.

Various implementations of RBE differ in the way the probabilities are represented and transformed in the process and measurement models. If the models are linear and the probabilities are Gaussian, the KF is an efficient and optimal solution in the least square sense. If the models are nonlinear (which is often the case in navigation systems), UKF or EKF [15] can be used. In EKF the models are linearized using Jacobian matrices, while in the UKF the probability distribution is approximated using a set of deterministically chosen (nonrandom sampling) points in the state space, which conserve the Gaussian properties of the distribution under nonlinear transformations [16]. The latter approach based on intuition that it is easier to approximate a probability distribution than to approximate an arbitrary nonlinear function or transformation.

Although historically EKF was a method of choice for solving navigational problems, the approach requires the first two terms of the Taylor series expansion to dominate the remaining terms. For some stronger nonlinearity the approach could lead to instability if the linearization assumption is violated. Although higher order versions of EKF also exist, their computational complexity makes them often unfeasible for practical usage in realtime applications and/or highly dimensional systems, and often a similar performance can be achieved with UKF. Note that the computational complexity of the UKF is of the same order as that of the EKF, but this only implies an asymptotic complexity and does not consider the scaling which can be significant in practical implementations.

\subsection{Sigma-Point Kalman Filtering}

Although the UKF algorithm is well-known and the details can be found elsewhere $[15,17]$, some non-trivial modifications are necessary for the presented IMU/GNSS/DVL filters. As we follow a full-state approach, an Augmented UKF configuration is employed, where the original state is augmented with noisy inertial sensor measurements in order to propagate them with the same accuracy as that of original variables of interest. A special care has to 
be taken regarding the attitude parametrization as unit attitude quaternions are deprived of one degree of freedom due to unit norm constraint [4].

The navigation filter is formulated as a nonlinear estimation problem for the system governed by the following stochastic models:

$$
\begin{gathered}
x_{k}=f\left(x_{k-1}, u_{k}, v_{k}\right), \\
z_{k}=h\left(x_{k}, \epsilon_{k}\right),
\end{gathered}
$$

where $u_{k}$ is the control input, $v_{k}$ is the zero mean process noise vector with covariance matrix $Q_{k}$ and $\epsilon_{k}$ is the observation noise vector with corresponding covariance matrix $R_{k}$. Within the presented work we evaluate several SPKF strategies, namely classical Unscented Kalman Filter (UKF), more efficient Spherical Simplex UKF (SUKF), CentralDifference KF (CDKF) and recently proposed Cubature KF (CKF). In all the cases we use fully-augmented SPKF, where the state is augmented with both process and measurement noises. Furthermore, special modifications are implemented in order to accommodate the attitude quaternion within the structure of SPKF. In the case of UKF one starts with initializing the filter with $\hat{x}_{0}=E\left[x_{0}\right]$ and the associated $P_{0}^{+}=E\left[\left(x_{0}-\hat{x}_{0}\right)\left(x_{0}-\hat{x}_{0}\right)^{T}\right]$. Then for every $k=1, \ldots, \infty$ one calculates the $\sigma$-points with $S_{k-1}^{a,+}\left(S_{k-1}^{a,+}\right)^{T}=P_{k-1}^{a,+}$ :

$$
\begin{aligned}
& \hat{x}_{k-1}^{a,+}=E\left[x_{k-1}^{a,+}\right]=\left[\begin{array}{lll}
\left(\hat{x}_{k-1}^{+}\right)^{T} & v_{k}^{T} & \epsilon_{k}^{T}
\end{array}\right]^{T}=\left[\begin{array}{ll}
\left(\hat{q}_{k-1}^{+}\right)^{T} & \left(\hat{x}_{k-1}^{a l q,+}\right)^{T}
\end{array}\right]^{T}, \\
& P_{k-1}^{a,+}=E\left[\left(x_{k-1}^{a,+}-\hat{x}_{k-1}^{a,+}\right)\left(x_{k-1}^{a,+}-\hat{x}_{k-1}^{a,+}\right)^{T}\right]=\left[\begin{array}{ccc}
P_{k-1}^{+} & 0 & 0 \\
0 & Q_{k} & 0 \\
0 & 0 & R_{k}
\end{array}\right], \\
& \mathcal{X}_{k-1}^{a,+}=\left[\begin{array}{c}
\mathcal{X}_{k-1}^{q,+} \\
\mathcal{X}_{k-1}^{a \mid q,+}
\end{array}\right]=\left[\begin{array}{ccc}
\hat{q}_{k-1}^{+} & \delta q_{1: n, k-1}^{+} \otimes \hat{q}_{k-1}^{+} & \left(\delta q_{1: n_{n}, k-1}^{+}\right)^{-1} \otimes \hat{q}_{k-1}^{+} \\
\hat{x}_{k-1}^{a l q,+} & \hat{x}_{k-1}^{a \backslash q,+}+\gamma S_{k-1}^{a l q,+} & \hat{x}_{k-1}^{a a q,+}-\gamma S_{k-1}^{a l q,+}
\end{array}\right],
\end{aligned}
$$

where $(\cdot)^{-1}$ is the quaternion inverse, $\otimes$ stands for the quaternion multiplication and $(\cdot)^{a \mid q}$ corresponds to the non-quaternion part of the state. In the expressions above:

$$
S_{k-1}^{a,+}=\left[\begin{array}{c}
S_{k-1}^{q,+} \\
S_{k-1}^{a l q,+}
\end{array}\right], \quad P_{k-1}^{a,+}=\left[\begin{array}{c}
P_{k-1}^{q,+} \\
P_{k-1}^{a \alpha q,+}
\end{array}\right], \quad \delta q_{i, k-1}^{+}=\left[\begin{array}{c}
\cos \left(\frac{\phi_{i, k-1}^{+}}{2}\right) \\
e_{i, k-1}^{+} \sin \left(\frac{\phi_{i, k-1}^{+}}{2}\right)
\end{array}\right],
$$


with rotation angle $\phi_{i, k-1}^{+}=\left|\gamma S_{k-1}^{q,+[i]}\right|$, rotation axis $e_{i, k-1}^{+}=S_{k-1}^{q,+[i]} /\left|S_{k-1}^{q,+[i]}\right|$ and with $(\cdot)^{[i]}$ meaning the $i$-th column of the matrix. The time-update equations with $n_{a}=n_{x}+n_{v}+n_{\epsilon}$ and barycentric mean for quaternion part of the state can be written as follows:

$$
\begin{gathered}
\mathcal{X}_{k}^{\chi,-}=f\left(\mathcal{X}_{k-1}^{x,}, u_{k}, \mathcal{X}_{k}^{v}\right), \\
\hat{x}_{k}^{-}=\sum_{i=0}^{2 n_{g}} w_{m}^{[i]} \mathcal{X}_{k}^{x,-,[i]},
\end{gathered}
$$

with subsequent quaternion normalization $\hat{q}_{k}^{-} \leftarrow \frac{\hat{q}_{k}^{-}}{\left|\hat{q}_{k}^{-}\right|}$and covariance:

$$
P_{k}^{-}=\left[\begin{array}{c}
P_{k}^{q,-} \\
P_{k}^{a \mid q,-}
\end{array}\right]=\left[\begin{array}{c}
\sum_{i=0}^{2 n_{q}} w_{c}^{[i]} \phi_{i, k}^{-} e_{i, k}^{-}\left(\phi_{i, k}^{-} e_{i, k}^{-}\right)^{T} \\
\sum_{i=0}^{2 n_{a}} w_{c}^{[i]}\left(\mathcal{X}_{k}^{\times \mid q,-,[i]}-\hat{x}_{k}^{-}\right)\left(\mathcal{X}_{k}^{x \mid q,-,[i]}-\hat{x}_{k}^{-}\right)^{T}
\end{array}\right],
$$

Where $\phi_{i, k}^{-}=2 \arccos \left(\left\lfloor\delta q_{i, k}^{-}\right\rfloor_{0}\right) \quad, \quad e_{i, k}^{-}=\left[\frac{\left\lfloor\delta q_{i, k}^{-}\right\rfloor_{1}}{\sqrt{1-\left\lfloor\delta q_{i, k}^{-}\right\rfloor_{0}^{2}}} \frac{\left\lfloor\delta q_{i, k}^{-}\right\rfloor_{2}}{\sqrt{1-\left\lfloor\delta q_{i, k}^{-}\right\rfloor_{0}^{2}}} \frac{\left\lfloor\delta q_{i, k}^{-}\right\rfloor_{3}}{\sqrt{1-\left\lfloor\delta q_{i, k}^{-}\right\rfloor_{0}^{2}}}\right]^{T}$, with $\delta q_{i, k}^{-}=\mathcal{X}_{i, k}^{q,-} \otimes\left(\hat{q}_{k}^{-}\right)^{-1}$ and $j$-th component selection operator from the quaternion.

The measurement update equations (assuming that there is no quaternion among the measurements):

$$
\begin{gathered}
\mathcal{Z}_{k}=h\left(\mathcal{X}_{k}^{x,-}, \mathcal{X}_{k}^{\epsilon}\right), \\
\hat{z}_{k}=\sum_{i=0}^{2 n_{g}} w_{m}^{[i]} \mathcal{Z}_{k}^{[i]}, \\
P_{z z, k}=\sum_{i=0}^{2 n_{a}} w_{c}^{[i]}\left(\mathcal{Z}_{k}^{[i]}-\hat{z}_{k}\right)\left(\mathcal{Z}_{k}^{[i]}-\hat{z}_{k}\right)^{T}, \\
P_{x z, k}=\left[\begin{array}{c}
\sum_{i=0}^{2 n_{a}} w_{c}^{[i]} \phi_{i, k}^{-} e_{i, k}^{-}\left(\mathcal{Z}_{k}^{[i]}-\hat{z}_{k}\right)^{T} \\
\sum_{i=0}^{2 n_{a}} w_{c}^{[i]}\left(\mathcal{X}_{k}^{x \mid q,-,[i]}-\hat{\chi}_{k}^{x \backslash q,-}\right)\left(\mathcal{Z}_{k}^{[i]}-\hat{z}_{k}\right)^{T}
\end{array}\right], \\
K_{k}=P_{x z, k} P_{z z z, k}^{-1}, \\
P_{k}^{+}=P_{k}^{-}-K_{k} P_{z z, k} K_{k}^{T},
\end{gathered}
$$

where: 


$$
\delta q_{k}^{+}=\left[\begin{array}{c}
\cos \left(\frac{\phi_{k}^{+}}{2}\right) \\
e_{k}^{+} \sin \left(\frac{\phi_{k}^{+}}{2}\right)
\end{array}\right],
$$

and $\phi_{k}^{+}=\left|K_{k}^{q}\left(z_{k}-\hat{z}_{k}\right)\right|$ with $e_{k}^{+}=\frac{K_{k}^{q}\left(z_{k}-\hat{z}_{k}\right)}{\left|K_{k}^{q}\left(z_{k}-\hat{z}_{k}\right)\right|}$. Some additional modifications are necessary if the quaternion is among the measurements. In the expressions above the parameter $\gamma=\sqrt{n_{a}+\lambda}$ and the corresponding weights:

$$
w_{m}^{[0]}=\frac{\lambda}{n_{a}+\lambda}, \quad w_{c}^{[0]}=\frac{\lambda}{n_{a}+\lambda}+\left(1-\alpha^{2}+\beta\right), \quad w_{m}^{[i]}=w_{c}^{[i]}=\frac{1}{2\left(n_{a}+\lambda\right)} .
$$

For $i=1, \ldots, 2 n_{a}, \lambda=\alpha^{2}\left(n_{a}+\kappa\right)-n_{a}$ being the compound scaling parameter, $1 e-4<\alpha \leq 1$ being the primary scaling parameter that determines how far the $\sigma$-points are spread from the mean, $\beta$ is the secondary scaling factor used to emphasize the weighting on the zeroth $\sigma$-point for covariance calculation and can be used to minimize certain higher order error terms based on known moments of the prior random variable. For Gaussian priors $\beta=2$ is optimal. The tertiary scaling factor $\kappa$ is often set to zero. Note that in general the optimal values of these parameters are problem specific. Finally, $\sqrt{ } \cdot$ is the matrix squareroot using lower triangular Cholesky decomposition and $n_{a}$ is the dimension of the augmented state vector $x_{k}^{a}$ with $x^{a}=\left[\begin{array}{lll}x^{T} & v^{T} & \epsilon^{T}\end{array}\right]^{T}$ and augmented $\sigma$-points are denoted $\mathcal{X}^{a}=\left[\begin{array}{lll}\left(\mathcal{X}^{x}\right)^{T} & \left(\mathcal{X}^{v}\right)^{T} & \left(\mathcal{X}^{\epsilon}\right)^{T}\end{array}\right]^{T}$. Note that the dimensionality of the quaternion is considered to be 3 , while the quaternion itself is a four-dimensional object.

One can see that attitude quaternion needs requires some special treatment when employed within a SPKF. An alternative strategy has been suggested in [22], where one considers the vector part of the quaternion for the covariance calculation assuming that the quaternion can be always properly reconstructed using the unit norm constraint. The required modifications are related to the calculation of $\sigma$-points in the prediction step (similar strategy can be employed if the $\sigma$-points have to be recalculated in the correction step of non-augmented SPKF), where the noise quaternions have to be calculated:

$$
\delta q_{i, k-1}^{+}=\left[\begin{array}{c}
\sqrt{1-\left(S_{k-1}^{q,+,[i]}\right)^{T}\left(S_{k-1}^{q,+,[i]}\right)} \\
S_{k-1}^{q,+,[i]}
\end{array}\right]
$$


Furthermore, the residuals for the quaternion part of the state are replaced simply by the vector part of the associated difference quaternion:

$$
\mathcal{X}_{k}^{q,-,[i]}-\hat{x}_{k}^{-} \rightarrow\left\lfloor\delta q_{i, k}^{-}\right\rfloor_{1: 3}
$$

Finally, the state update of the quaternion is formulated as:

$$
\delta q_{k}^{+}=\left[\begin{array}{c}
\sqrt{1-\left(\zeta_{k}^{+}\right)^{T}\left(\zeta_{k}^{+}\right)} \\
\left(\zeta_{k}^{+}\right)
\end{array}\right],
$$

where $\zeta_{k}^{+}=K_{k}^{q}\left(z_{k}-\hat{z}_{k}\right)$. The presented idea models the quaternion noise as a vector part of the quaternion which leads to the orientation uncertainty in the attitude. Then the noise vector is simply expanded into the quaternion parametrization using positive values of the scalar part of the quaternion. Clearly, as the quaternion process noise is a 3-dimensional noise vector, it is expanded into a 4D unit quaternion to apply the multiplicative quaternion error approach.

The extension of the presented UKF approach to other SPKF strategies is rather straightforward. As the computational demand of the UKF is strongly dependent on the number of $\sigma$-points used to represent the distribution, one can try to use a more efficient SUKF configuration with only $n_{a}+2$ points used to represent the distribution [17]. Here one chooses the initial weight $0 \leq W_{0} \leq 1$ with the sequence of weights calculated as $W_{i}=\left(1-W_{0}\right) /\left(n_{a}+1\right), i=1, \ldots, n_{a}+1$. For the scaled transformation (similarly as in the case of classical UKF) the previous weights are transformed as:

$$
w_{i}= \begin{cases}1+\left(W_{0}-1\right) / \alpha^{2}, & i=0, \\ W_{i} / \alpha^{2}, & i \neq 0 .\end{cases}
$$

Then the set of prototype $\sigma$-points $\mathcal{Y}^{[i]}$ by initializing:

$$
\mathcal{Y}_{0}^{1}=[0], \quad \mathcal{Y}_{1}^{1}=\left[-\frac{1}{\sqrt{2 w_{1}}}\right], \quad \mathcal{Y}_{2}^{1}=\left[\frac{1}{\sqrt{2 w_{1}}}\right] .
$$

Here $\mathcal{Y}_{i}^{j}$ is the $i$-th $\sigma$-point in the set for the $j$ th dimensional space. The corresponding vector sequence is expanded for $j=2, \ldots, n_{a}$ as explained in details in [17]. The rest of the procedure is fairly similar to the one of classical UKF with:

$$
\mathcal{X}_{k-1}^{a,+}=\left[\begin{array}{c}
\mathcal{X}_{k-1}^{q,+} \\
\mathcal{X}_{k-1}^{a \backslash q,+}
\end{array}\right]=\left[\begin{array}{c}
\delta q_{1: n_{a}, k-1}^{+} \otimes \hat{q}_{k-1}^{+} \\
\hat{x}_{k-1}^{a \backslash q,+}+\left(S_{k-1}^{a,-} \mathcal{Y}\right)^{a \backslash q}
\end{array}\right] .
$$


Similar strategy can be applied to CDKF [23] with quaternion attitude parametrization, where the covariance calculation involves $2^{\text {nd }}$ order differences of the associated $\sigma$-points.

Finally, the results are presented for a recently presented so-called Cubature Kalman Filter [24]. In this filter each $\sigma$-point $\mathcal{X}_{k-1}^{a,+[i]}$ is calculated using the prototype cubature points $\xi_{n_{a}}^{[i]}$ :

$$
\mathcal{X}_{k-1}^{a,+,[i]}=\left[\begin{array}{c}
\mathcal{X}_{k-1}^{q,+,[i]} \\
\mathcal{X}_{k-1}^{a \mid q,+,[i]}
\end{array}\right]=\left[\begin{array}{c}
\delta q_{i, k-1}^{+} \otimes \hat{q}_{k-1}^{+} \\
\hat{X}_{k-1}^{a \mid q,+}+\left(S_{k-1}^{a,+} \xi_{n_{a}}^{[i]}\right)^{a \backslash q}
\end{array}\right], \quad i=0, \ldots, 2 n_{a}-1 .
$$

The prototype cubature points of the dimensionality $n_{a}$ are defined as:

$$
\xi_{n_{a}}^{[i]}=\sqrt{n_{a}}[1]_{n_{a}}^{[i]}, \quad i=0, \ldots, 2 n_{a}-1, \quad n_{a}=n_{x}+n_{\nu} .
$$

For example, a set of points for $\xi_{2}$ is $\left\{\left(\begin{array}{ll}1 & 0\end{array}\right)^{T},\left(\begin{array}{ll}0 & 1\end{array}\right)^{T},\left(\begin{array}{ll}-1 & 0\end{array}\right)^{T},\left(\begin{array}{ll}0 & -1\end{array}\right)^{T}\right\}$ with the generator point $\left(\begin{array}{ll}1 & 0\end{array}\right)^{T}$.

A clear advantage of the SPKF schemes is that direct process and measurement models can be used and no intricate Jacobians have to be calculated. Finally, the SPKFs are able to preserve higher order statistical information compared to de facto engineering standard EKF and have shown superior performance for some highly nonlinear problems.

\subsection{Dynamical Models}

As a process model we employ a classical strapdown inertial mechanization with unit quaternion $q=\left[\begin{array}{llll}q_{1} & q_{2} & q_{3} & q_{4}\end{array}\right]^{T}$ for attitude representation, where the quaternion kinematics is obtained from:

$$
\dot{q}=\frac{1}{2} \Omega(\omega) q, \quad \Omega(\omega)=\left[\begin{array}{cc}
0 & -\omega^{T} \\
\omega & -[\omega \times]
\end{array}\right],
$$

and cross product matrix $[\omega \times]$. The discrete equivalent is obtained using trapezoidal integration with:

$$
\Omega\left(\omega_{k}^{B}\right)=\Omega\left(\tilde{\omega}_{k}^{B}-\hat{b}_{G, k}-C_{E}^{B}\left(\hat{q}_{k}\right) \omega_{I E}\right),
$$

where $\tilde{\omega}_{k}^{B}$ is the angular rate measured in body frame, $\hat{b}_{G, k}$ is the estimate of the gyroscope bias, $\omega_{I E}$ is the Earth rotation rate with $C_{E}^{B}\left(\hat{q}_{k}\right)$ being the rotation matrix from ECEF to Body calculated from the quaternion estimate $\hat{q}_{k}$. Similar bias compensation has to be performed for the accelerometer signal before strapdown inertial mechanization. The 
rest of the process model implementation follows classical strapdown mechanization in ECEF frame. The state of the filter consists of the attitude quaternion, position and velocity (both in ECEF) as well as both gyroscope and accelerometer offsets. For tightly-coupled filters both GNSS receiver clock and clock offset rate have to be added to the filter state.

There several options to constructing the measurement models depending on the configuration of the filter. For loosely-coupled approaches a snapshot least-square solution is used for both position and velocity [19] or corresponding RTK solution is taken (e.g. from RTKLIB [20]). Within the tightly-coupled schemes one assumes direct observation models for both code and Doppler shift measurement using essentially the same mathematics as adopted in corresponding snapshot solutions. Obviously, for both types of raw GNSS observations (C/A L1 pseudoranges and Doppler shifts) lever arm compensations have to be implemented, although for latter type of measurements the lever arm can be often ignored due to relatively low dynamics of a typical commercial vessel.

The speed log measurement model ( $X-Y$ velocity measured in vessel frame) can be written as follows:

$$
\tilde{z}_{S L, k}^{V}=C_{B}^{V} \cdot C_{E}^{B}\left(\hat{q}_{k}\right)\left(\hat{v}_{k}^{E}+C_{B}^{E}\left(\hat{q}_{k}\right) \Omega\left(\omega_{k}^{B}\right) r_{S L}^{B}\right),
$$

where $V$ is the DVL coordinate frame with $r_{S L}^{B}$ being the lever arm with respect to IMU. Note that classical IMU/GNSS approach does not impose any non-holonomic constraints in $X Y$ plane (e.g. that vessel is able to move only in the direction of heading) or similar. The alternative is to employ the constraint along the body vertical axis of the vessel (velocity projection in the body frame) as one can assume the vertical velocity to be on average zero. The constraint can be implemented within the KF framework as so-called "pseudo-measurement" by extending the model from above for the third component and setting the measurement to zero with some associated modeling noise. Although this is able to decrease significantly the vertical position drift, the trick could introduce modeling errors and correlated measurement noise and, therefore, the validity of the approach has to be carefully investigated using real measurement data.

Obviously, for lower-cost MEMS IMU the navigation performance is strongly degraded due to fast accumulation of the errors caused by sensor noises, biases, scale factor errors, etc. Moreover, for non-augmented IMU/GNSS system (e.g. a system without the magnetometer, gyrocompass or multiple GNSS antennas), the attitude and some of the inertial sensor errors become weakly observable and their observability is strongly 
conditioned on the dynamics of the vessel. Due to these reasons it has been decided to incorporate the baseline observations (non-collinear vector observations) from available three spatially distributed GNSS antennas to ensure that the attitude drift is constrained when baseline measurements are available. The baseline observation is considered to valid if both antennas have RTK position fix and therefore from 0 (e.g. none) to 3 baseline observations can be incorporated into the measurement model on the rate of their availability. The advantage of direct baseline vector observation model is that heading becomes observable even with a single observation of non-vertical baseline.

\section{Setup}

In order to overcome the previously identified issues and to commit with the IMO requirements, the DLR has developed a PNT unit concept [2] and an operational prototype in order to confirm the PNT unit performance under real operational conditions. Here the core goals are the provision of redundancy by support of all on-board PNT relevant sensor data including Differential GNSS (DGNSS) and future possible backup systems (e.g., eLoran), the design and implementation of parallel processing chains (single-sensor and multi-sensor architectures) for robust PNT data provision and the development of both multi-sensor fusion and the associated integrity algorithms.

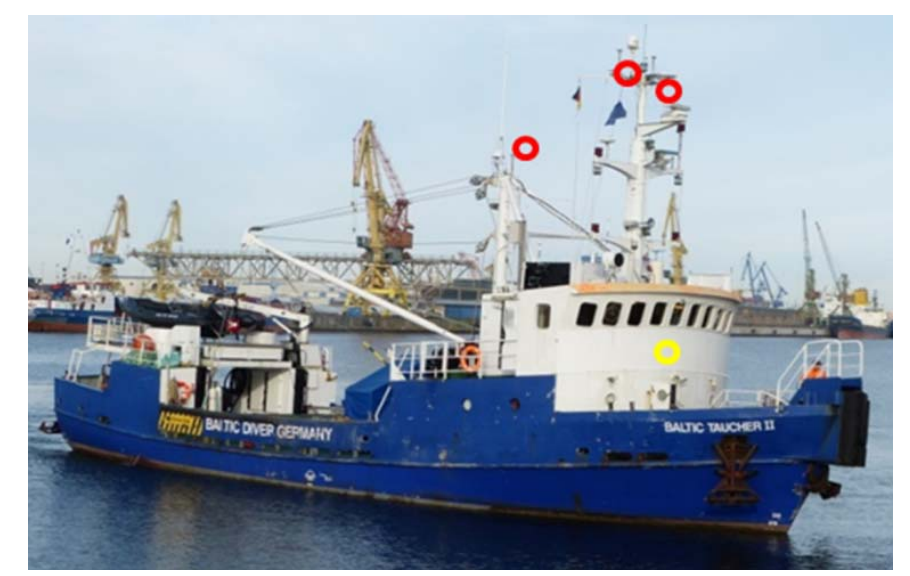

Figure 1. Baltic Taucher II test vessel. Yellow circle represents the IMU placement and the red circles stand for GNSS antenna positions (3x).

The sensor measurements were recorded using the multipurpose research and diving vessel Baltic Diver II (length 29 m, beam 6.7 m, draught 2.8 m, GT 146 t). The vessel was equipped with three dual frequency GNSS antennas (forming almost isosceles triangle with corresponding sides of $5.27 \mathrm{~m}, 5.17 \mathrm{~m}$ and $1.26 \mathrm{~m}$ and Antenna 1 being placed in front of the vessel with altitude $2.46 \mathrm{~m}$ higher, see Figure 1.) and receivers (Javad Delta), a fiber-optic gyroscope (FOG) IMU (iMar IVRU FCAI), gyrocompass, DVL and echo 
sounder. Additionally a MEMS IMU module was developed based on tactical grade IMU (Analog Devices ADIS 16485) and commercial ARM-based embedded platform. Both FOG and MEMS IMUs are sampled at $200 \mathrm{~Hz}$. For the velocity measurements Furuno Doppler Sonar DS-60 was employed. The sonar is fully compliant with IMO MSC.36(63), MSC.96(72), A.694(17) and A.824(19), required for the vessels of 50,000 GT and greater and is able to deliver the precise measurements suitable for berthing and docking maneuvers.

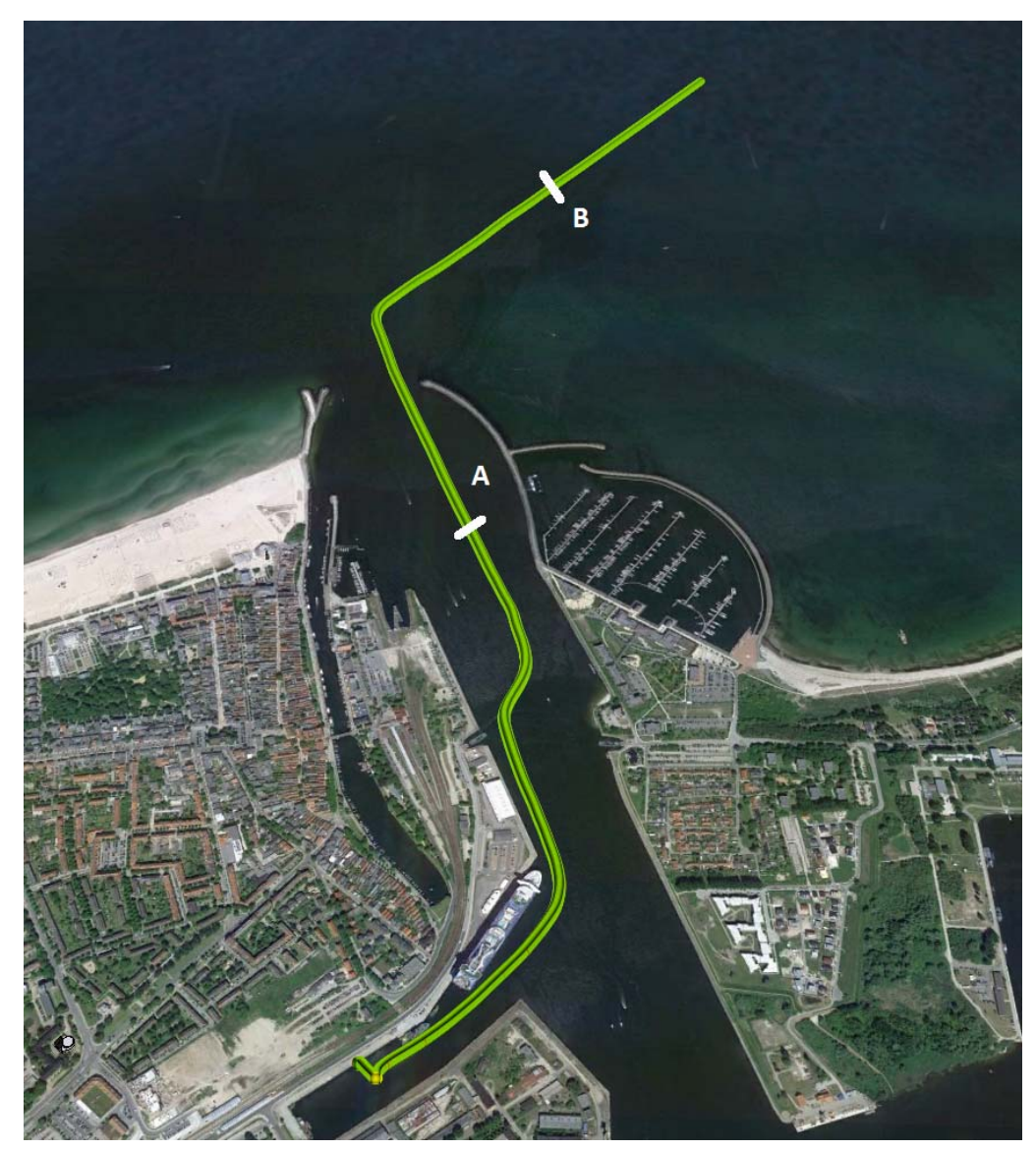

Figure 2. The test trajectory (approx. $22 \mathrm{~min}$ ) in the port of Rostock (overlaid with the image from Google Earth). Segment AB denotes the path where GNSS signals were disabled (5 minutes).

The IALA (International Association of Marine Aids to Navigation and Lighthouse Authorities) beacon antenna and receiver were employed for the reception of the IALA DGNSS code corrections. The VHF modem was configured for the reception of RTK corrections data from Maritime Ground Based Augmentation System (MGBAS) station located in the port of Rostock. The MGBAS reference station provides GPS code and phase corrections with $2 \mathrm{~Hz}$ update rate for both $\mathrm{L} 1$ and $\mathrm{L} 2$ frequencies. These correction data are used for a highly accurate RTK positioning (reference) on board the vessel. All the relevant sensor measurements are provided either directly via Ethernet or via serial to 
Ethernet adapter to a Box PC where the observations are processed in real-time and stored in a SQlite3 database. The described setup enables a record and replay functionality for further processing of the original sensor data.

\section{Results}

In order to evaluate the performance of the proposed hybrid navigation system we have used real measurements (see Figure 2.) from the operating vessel in the port of Rostock (Germany) and simulating the GNSS outage of 5 minutes by immediately disabling all the satellites including the GNSS compass functionality. Although more advanced scenarios could include the satellites disappearing one by one, this would make the analysis far more complicated as the performance of the navigation filter would depend on the order how the satellites are jammed and re-acquired. The initial data segment of approx. 13 minutes is left undisturbed in order for the filter to converge.

The filters were implemented assuming measurement noise of 5 meters for code measurement (pseudorange), Doppler velocity measurement noise of $0.2 \mathrm{~m} / \mathrm{s}$, RTK position solution noise of $0.05 \mathrm{~m}$ and RTK velocity solution noise of $0.1 \mathrm{~m} / \mathrm{s}$ (circular covariance approximation). In order for the analysis to be fair, we have paid a special attention to the equivalent noise mapping between the corresponding loosely- and tightlycoupled solutions. Clearly, the constant circular covariance model is often not a good approximation with respect to particular satellite geometry (matrix $G$ ) with effective measurement noise covariance of the snapshot solution:

$$
R_{L C}=\left(G^{T} R_{P R}^{-1} G\right)^{-1}
$$

where $R_{P R}$ is the is the corresponding covariance of the pseudorange measurements, while still circular covariance of $1 \mathrm{~cm} / \mathrm{s}$ was assumed for snapshot Doppler solution. The GNSS compass baseline noise was assumed to be $5 \mathrm{~cm}$ per component of the vector. The process noise values were correspondingly tuned to the specification of inertial sensors (ADIS16485 and iMar ilMU FCAI) with the clock process noise adjusted to the observed dynamics of the GNSS receiver. The DVL noise was set slightly higher than the datasheet specification in order to accommodate possible modeling errors such as DVL misalignment, scale factor errors etc. The measurement noise for both $X$ and $Y$ axis was set to $30 \mathrm{~cm} / \mathrm{s}$, while the $Z$ axis pseudo-measurement noise was set to $1 \mathrm{~m} / \mathrm{s}$. Such large mismatch is caused by the fact that, in principle, the vessel is actually moving in vertical direction due to waves and this would result in violation of the noise assumptions due to 
correlations in the residual statistics and the inflated measurement noise is the simplest approach to reduce the impact of such correlated noise on the estimated state.

Table 1: HPE and VPE performance of different IMU/GNSS/DVL integration schemes for 5 minutes GNSS outage (LC - loosely-coupled, TC - tightly-coupled).

\begin{tabular}{|c|c|c|c|c|c|c|c|c|}
\hline & \multicolumn{2}{|c|}{$\begin{array}{c}\text { IMU/GNSS } \\
\text { DVL (2D + 1D) }\end{array}$} & \multicolumn{2}{c|}{$\begin{array}{c}\text { IMU/GNSS } \\
\text { no DVL }\end{array}$} & \multicolumn{2}{c|}{$\begin{array}{c}\text { IMU/GNSS } \\
\text { DVL (2D) }\end{array}$} & \multicolumn{2}{c|}{$\begin{array}{c}\text { IMU/GNSS } \\
\text { DVL (1D) }\end{array}$} \\
\hline & HPE, [m] & VPE, [m] & HPE, [m] & VPE, [m] & HPE, [m] & VPE, [m] & HPE, [m] & VPE, [m] \\
\hline $\begin{array}{c}\text { LC IMU/GNSS/DVL: } \\
\text { FOGG + RTK }\end{array}$ & 19.55 & $\mathbf{5 . 2 6}$ & 431.60 & 10.97 & 19.55 & 65.29 & 299.73 & 8.40 \\
\hline $\begin{array}{c}\text { LC IMU/GNSS/DVL: } \\
\text { FOG + SPP }\end{array}$ & $\mathbf{1 6 . 5 3}$ & $\mathbf{7 . 1 9}$ & 676.07 & 25.99 & 16.50 & 54.20 & 496.11 & 12.72 \\
\hline $\begin{array}{c}\text { TC IMU/GNSS/DVL: } \\
\text { FOG }\end{array}$ & $\mathbf{1 7 . 2 7}$ & $\mathbf{7 . 3 4}$ & 679.51 & 11.33 & 17.19 & 52.98 & 494.75 & 13.17 \\
\hline $\begin{array}{c}\text { LC IMU/GNSS/DVL: } \\
\text { MEMS + RTK }\end{array}$ & $\mathbf{1 7 . 2 6}$ & $\mathbf{8 . 0 3}$ & $2.92 \mathrm{e}+03$ & 94.80 & 17.23 & 45.62 & 816.99 & 51.41 \\
\hline $\begin{array}{c}\text { LC IMU/GNSS/DVL: } \\
\text { MEMS + SPP }\end{array}$ & $\mathbf{1 4 . 7 1}$ & $\mathbf{9 . 8 4}$ & $2.34 \mathrm{e}+03$ & 72.36 & 14.75 & 47.02 & 951.57 & 56.08 \\
\hline $\begin{array}{c}\text { TC IMU/GNSS/DVL: } \\
\text { MEMS }\end{array}$ & $\mathbf{1 7 . 7 2}$ & $\mathbf{9 . 9 2}$ & $2.35 \mathrm{e}+03$ & 97.57 & 14.74 & 46.23 & 949.83 & 56.64 \\
\hline
\end{tabular}

Table 1 presents the results on bridging the GNSS outage of approx. 5 minutes using different measurement model configurations and filter structures (loosely-coupled with snapshot solution (SPP, both position and velocity), loosely-coupled with RTK (both position and velocity) solution and tightly-coupled approaches) and different quality of IMU (lower performance MEMS ADIS and higher performance FOG IMAR). The performance of the methods was assessed by considering correspondingly maximal horizontal position error (HPE) and vertical position error (VPE) during the GNSS outage with respect to the reference trajectory where no GNSS outage was imposed. In order to evaluate the benefit of using DVL for autonomous navigation we have considered a classical pure IMU/GNSS configuration (no DVL), IMU/GNSS with true 2D DVL measurements, IMU/GNSS with only 1D $Z$ axis vertical velocity constraint and, finally, IMU/GNSS/DVL with both 2D real $X, Y$ measurements and associated $Z$ axis motion constraint. All the filters employed GNSS compass baseline measurements (except of GNSS outage segment) as this is critical to ensure the attitude observability in the case of IMU/GNSS systems with reduced dynamics. The corresponding results for the time needed for the algorithm to accumulate 
HPE of correspondingly 10 meters and 25 meters are shown in Table 2. An intuitive view of both HPE and VPE growth with time can be found correspondingly in Figure 3 for different filter architectures and in Figure 4 for different DVL measurement models.

Table 2: Performance of different IMU/GNSS/DVL integration schemes in terms of time needed to reach 10 meter and 25 meter HPE (LC - loosely-coupled, TC - tightly-coupled).

\begin{tabular}{|c|c|c|c|c|c|c|c|c|}
\hline & \multicolumn{2}{|c|}{$\begin{array}{c}\text { IMU/GNSS } \\
\text { DVL (2D + 1D) }\end{array}$} & \multicolumn{2}{|c|}{$\begin{array}{l}\text { IMU/GNSS } \\
\text { no DVL }\end{array}$} & \multicolumn{2}{|c|}{$\begin{array}{l}\text { IMU/GNSS } \\
\text { DVL (2D) }\end{array}$} & \multicolumn{2}{|c|}{$\begin{array}{l}\text { IMU/GNSS } \\
\text { DVL (1D) }\end{array}$} \\
\hline & $\begin{array}{l}\text { Time to } \\
\text { HPE 10m, } \\
\text { [sec] }\end{array}$ & $\begin{array}{l}\text { Time to } \\
\text { HPE 25m, } \\
\text { [sec] }\end{array}$ & $\begin{array}{l}\text { Time to } \\
\text { HPE 10m, } \\
\text { [sec] }\end{array}$ & $\begin{array}{l}\text { Time to } \\
\text { HPE 25m, } \\
\text { [sec] }\end{array}$ & $\begin{array}{l}\text { Time to } \\
\text { HPE 10m, } \\
\text { [sec] }\end{array}$ & $\begin{array}{l}\text { Time to } \\
\text { HPE 25m, } \\
\text { [sec] }\end{array}$ & $\begin{array}{l}\text { Time to } \\
\text { HPE 10m, } \\
\text { [sec] }\end{array}$ & $\begin{array}{l}\text { Time to } \\
\text { HPE 25m, } \\
\text { [sec] }\end{array}$ \\
\hline $\begin{array}{l}\text { LC IMU/GNSS/DVL: } \\
\text { FOG + RTK }\end{array}$ & 140 & - & 88 & 126 & 140 & - & 87 & 124 \\
\hline $\begin{array}{c}\text { LC IMU/GNSS/DVL: } \\
\text { FOG + SPP }\end{array}$ & 137 & - & 72 & 107 & 137 & - & 72 & 106 \\
\hline $\begin{array}{c}\text { TC IMU/GNSS/DVL: } \\
\text { FOG }\end{array}$ & 135 & - & 72 & 107 & 135 & - & 73 & 107 \\
\hline $\begin{array}{l}\text { LC IMU/GNSS/DVL: } \\
\text { MEMS + RTK }\end{array}$ & 147 & - & 42 & 57 & 147 & - & 42 & 57 \\
\hline $\begin{array}{l}\text { LC IMU/GNSS/DVL: } \\
\text { MEMS + SPP }\end{array}$ & 140 & - & 47 & 64 & 141 & - & 47 & 64 \\
\hline $\begin{array}{c}\text { TC IMU/GNSS/DVL: } \\
\text { MEMS }\end{array}$ & 140 & - & 46 & 63 & 141 & - & 47 & 64 \\
\hline
\end{tabular}

The corresponding HPEs and VPEs for different SPKF schemes are shown in Table 3 with the associated time needed to reach the 10 meter and 25 meter HPE provided in Table 4. The time evolution of both HPE and VPE for tightly-coupled FOG-based IMU/GNSS/DVL and different SPKF schemes is presented in Figure 5. Note that in this case the tightlycoupled architecture provides the most interesting behavior compared to simpler looselycoupled approaches, where all the SPKFs are performing relatively similarly as one can see in Tables 3 and 4.

Finally, Table 5 provides a comparison between two different approaches of representing the attitude quaternion noise within a SPKF (in this case classical symmetric UKF). While the first approach adopts a strategy where the attitude noise is represented via an equivalent rotation vector calculated from the difference quaternion (attitude residual), the second approach assumes the attitude noise to be represented by the vector part of the corresponding difference quaternion [22]. 
Horizontal Position Error for IMU/GNSS/DVL (UKF, 5 min. GNSS outage)

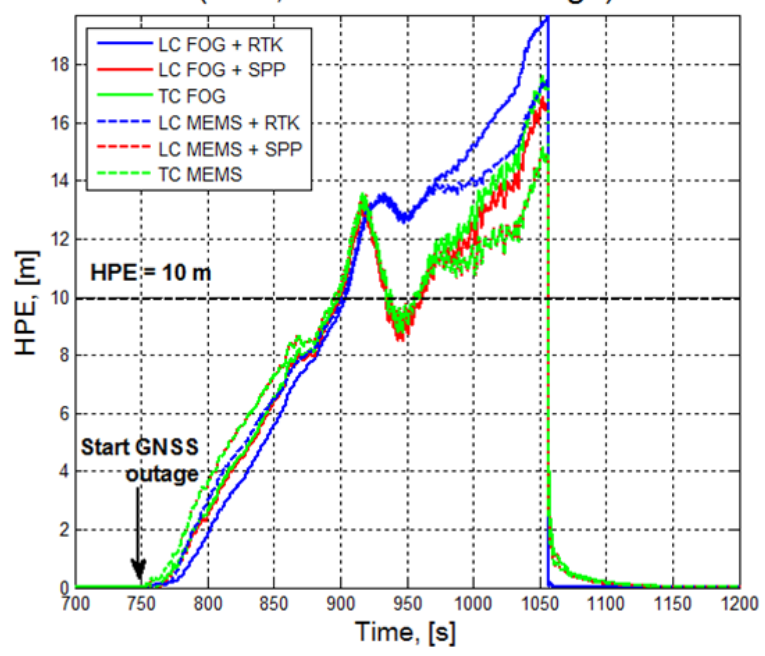

Vertical Position Error for IMU/GNSS/DVL (UKF, 5 min. GNSS outage)

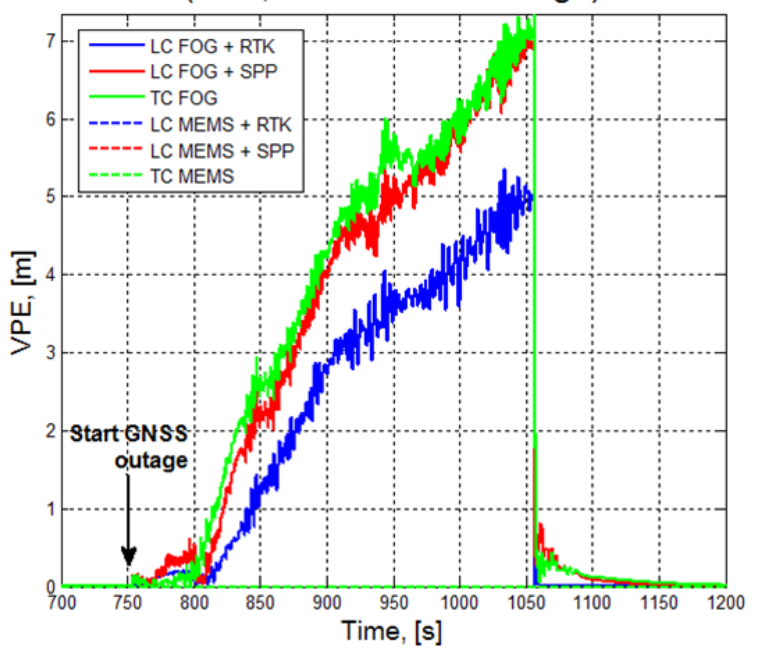

Figure 3. Horizontal position error (left) and vertical position error (right) during 5 minutes GNSS outage for different configurations of IMU/GNSS/DVL using complete (3D) DVL measurement model.

Horizontal Position Error for IMU/GNSS (TC, UKF, FOG (IMAR) IMU, 5 min. GNSS outage)

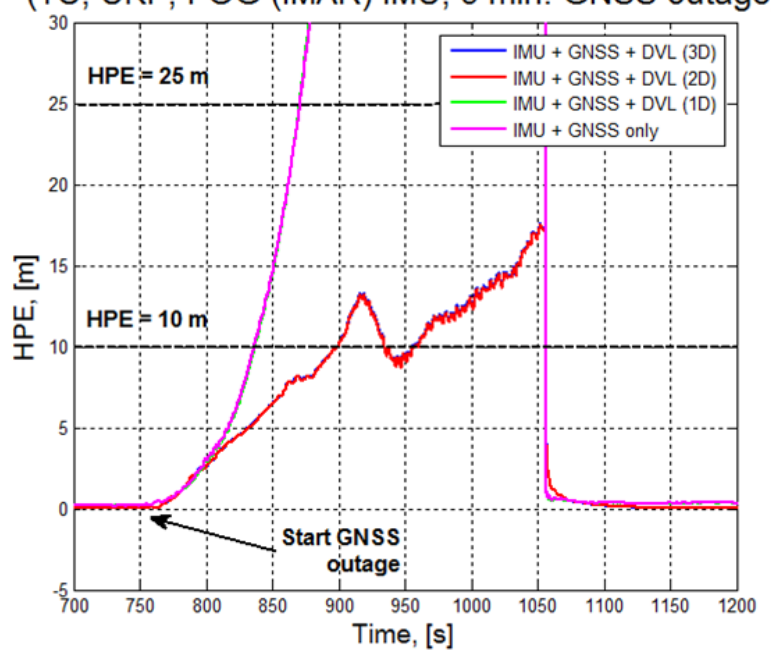

Vertical Position Error for IMU/GNSS (TC, UKF, FOG (IMAR) IMU, 5 min. GNSS outage)

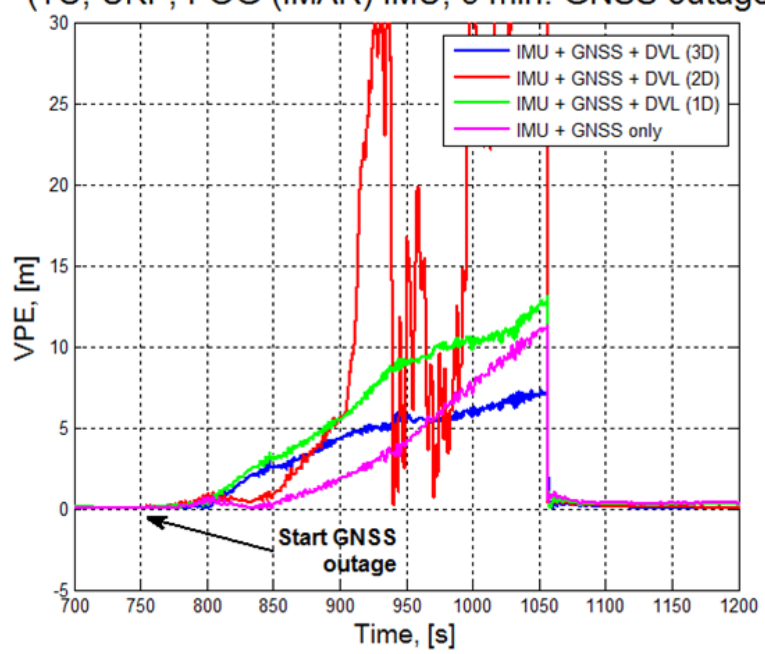

Figure 4. Horizontal position error (left) and vertical position error (right) during 5 minutes GNSS outage for tightly-coupled IMU/GNSS/DVL integration using different DVL measurement models. 
Horizontal Position Error for IMU/GNSS (different SPKFs) Vertical Position Error for IMU/GNSS (different SPKFs)
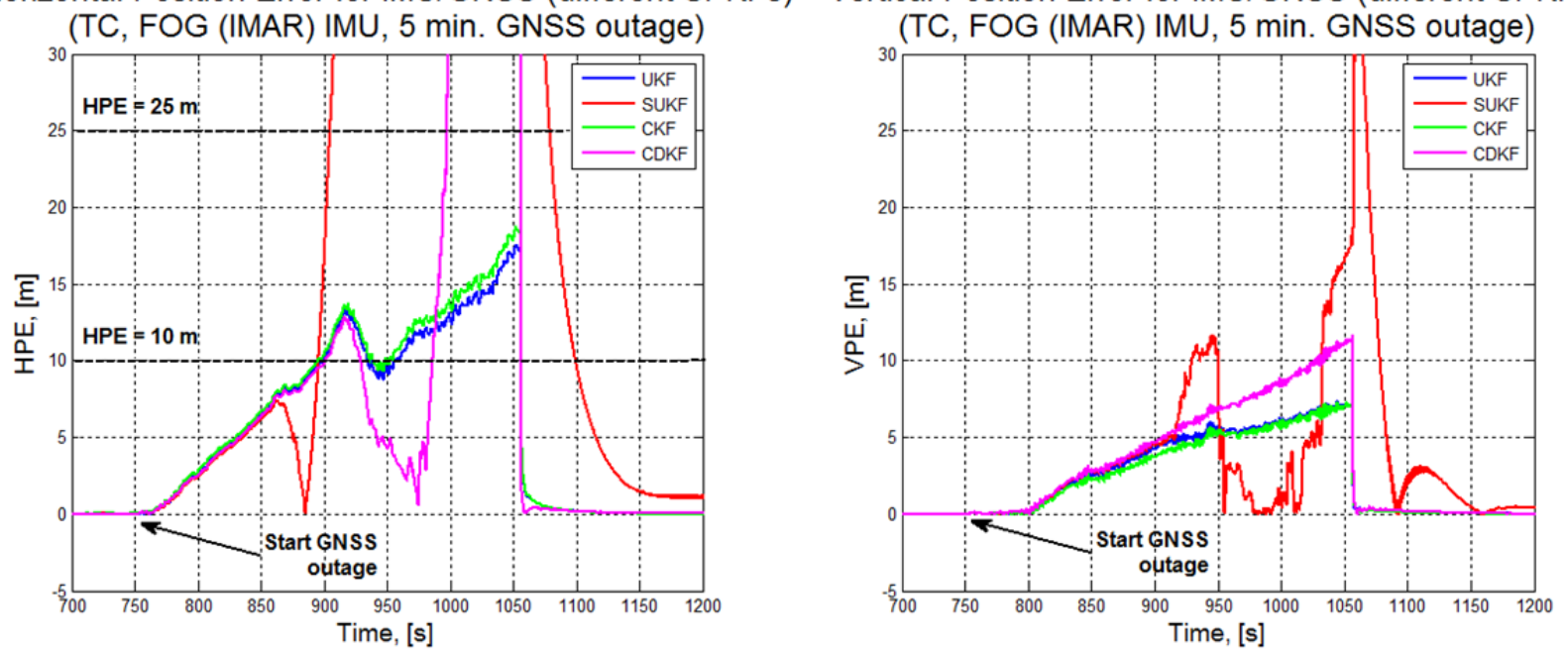

Figure 5. Horizontal position error (left) and vertical position error (right) during 5 minutes GNSS outage for tightly-coupled FOG-based IMU/GNSS/DVL integration using different SPKF schemes.

Table 3: Performance of IMU/GNSS/DVL integration schemes using different SPKF methods in terms of HPE and VPE (LC - loosely-coupled, TC - tightly-coupled).

\begin{tabular}{|c|c|c|c|c|c|c|c|c|}
\hline & \multicolumn{2}{|c|}{$\begin{array}{l}\text { Unscented KF } \\
\text { (UKF) }\end{array}$} & \multicolumn{2}{|c|}{$\begin{array}{l}\text { Spherical Simplex } \\
\text { Unscented KF } \\
\text { (SUKF) }\end{array}$} & \multicolumn{2}{|c|}{$\begin{array}{c}\text { Central-Difference } \\
\text { KF (CDKF) }\end{array}$} & \multicolumn{2}{|c|}{ Cubature KF (CKF) } \\
\hline & HPE, [m] & VPE, [m] & HPE, [m] & VPE, [m] & HPE, [m] & VPE, [m] & HPE, [m] & VPE, [m] \\
\hline $\begin{array}{c}\text { LC IMU/GNSS/DVL: } \\
\text { FOG + RTK }\end{array}$ & 19.55 & 5.26 & 21.01 & 5.97 & 20.10 & 5.41 & 19.35 & 5.52 \\
\hline $\begin{array}{c}\text { LC IMU/GNSS/DVL: } \\
\text { FOG + SPP }\end{array}$ & 16.53 & 7.19 & 16.15 & 7.83 & 16.41 & 7.60 & 16.47 & 7.47 \\
\hline $\begin{array}{c}\text { TC IMU/GNSS/DVL: } \\
\text { FOG }\end{array}$ & 17.27 & 7.34 & 631.12 & 17.64 & 81.84 & 11.67 & 18.44 & 7.31 \\
\hline $\begin{array}{l}\text { LC IMU/GNSS/DVL: } \\
\text { MEMS + RTK }\end{array}$ & 17.26 & 8.03 & 34.51 & 8.72 & 17.17 & 8.36 & 17.36 & 8.17 \\
\hline $\begin{array}{l}\text { LC IMU/GNSS/DVL: } \\
\text { MEMS + SPP }\end{array}$ & 14.71 & 9.84 & 33.06 & 12.42 & 16.69 & 10.02 & 14.79 & 10.00 \\
\hline $\begin{array}{c}\text { TC IMU/GNSS/DVL: } \\
\text { MEMS }\end{array}$ & 17.72 & 9.92 & 582.29 & 8.90 & 94.20 & 12.95 & 14.80 & 10.10 \\
\hline
\end{tabular}


Table 4: Performance of IMU/GNSS/DVL integration schemes in terms of time needed to reach 10 meter and 25 meter HPE (LC - loosely-coupled, TC - tightly-coupled).

\begin{tabular}{|c|c|c|c|c|c|c|c|c|}
\hline & \multicolumn{2}{|c|}{$\begin{array}{c}\text { Unscented KF } \\
\text { (UKF) }\end{array}$} & \multicolumn{2}{|c|}{$\begin{array}{c}\text { Spherical Simplex } \\
\text { Unscented KF } \\
\text { (SUKF) }\end{array}$} & \multicolumn{2}{|c|}{$\begin{array}{c}\text { Central-Difference } \\
\text { KF (CDKF) }\end{array}$} & \multicolumn{2}{c|}{ Cubature KF (CKF) } \\
\hline & $\begin{array}{c}\text { Time to } \\
\text { HPE 10m, } \\
{[\mathrm{sec}]}\end{array}$ & $\begin{array}{c}\text { Time to } \\
\text { HPE 25m, } \\
\text { [sec] }\end{array}$ & $\begin{array}{c}\text { Time to } \\
\text { HPE 10m, } \\
{[\mathrm{sec}]}\end{array}$ & $\begin{array}{c}\text { Time to } \\
\text { HPE 25m, } \\
\text { [sec] }\end{array}$ & $\begin{array}{c}\text { Time to } \\
\text { HPE 10m, } \\
\text { [sec] }\end{array}$ & $\begin{array}{c}\text { Time to } \\
\text { HPE 25m, } \\
\text { [sec] }\end{array}$ & $\begin{array}{c}\text { Time to } \\
\text { HPE 10m, } \\
\text { [sec] }\end{array}$ & $\begin{array}{c}\text { Time to } \\
\text { HPE 25m, } \\
\text { [sec] }\end{array}$ \\
\hline $\begin{array}{c}\text { LC IMU/GNSS/DVL: } \\
\text { FOG + RTK }\end{array}$ & 140 & - & 135 & - & 139 & - & 141 & - \\
\hline $\begin{array}{c}\text { LC IMU/GNSS/DVL: } \\
\text { FOG + SPP }\end{array}$ & 137 & - & 138 & - & 137 & - & 137 & - \\
\hline $\begin{array}{c}\text { TC IMU/GNSS/DVL: } \\
\text { FOG }\end{array}$ & 135 & - & 132 & 141 & 137 & 233 & 131 & - \\
\hline $\begin{array}{c}\text { LC IMU/GNSS/DVL: } \\
\text { MEMS + RTK }\end{array}$ & 147 & - & 137 & 272 & 149 & - & 147 & - \\
\hline $\begin{array}{c}\text { LC IMU/GNSS/DVL: } \\
\text { MEMS + SPP }\end{array}$ & 140 & - & 133 & 284 & 140 & - & 140 & - \\
\hline $\begin{array}{c}\text { TC IMU/GNSS/DVL: } \\
\text { MEMS }\end{array}$ & 140 & - & 138 & 148 & 144 & 239 & 140 & - \\
\hline
\end{tabular}

\section{Discussion}

The results shown in Table I clearly indicate that all configurations of full IMU/GNSS/DVL solutions allow the system to navigate without GNSS for extended period of time with reasonable accuracy. Interestingly, the difference between systems based on MEMS and FOG IMU is rather marginal. In contrary, for pure IMU/GNSS system the GNSS outage of 5 minutes can be considered too long for the required HPE less than 10 meters. Although the performance of the pure inertial bridging can be still somehow improved by setup calibration (e.g. GNSS compass geometry, IMU calibration, etc.) and corresponding filter parameter tuning, bridging of the GNSS outages with the duration longer than 10 minutes does not seem to be feasible, at least in foreseeable future and for IMUs of reasonable price. Still, for pure inertial bridging one clearly sees how the IMU performance affects the position errors as the errors of the FOG-system are significantly smaller compared to those of MEMS-based approach. Although the HPE performance of the 2D DVL measurement model is extremely similar to that of $3 D$, the vertical position error seems to be significantly larger even when compared to pure inertial approach. We believe that that could be explained by the modeling errors of the DVL which propagate to both the inertial sensor errors and vertical velocity. Finally, although the sole purpose of DVL 1D model 
was to constraint the vertical position drift, one can still observe an improvement in terms of HPE. Note that the results of pure inertial integration for FOG IMU can be hardly considered representative (due to filter convergence, time, offset dynamics and setup errors) and should be analyzed only relative to those of DVL-augmented systems as all the numbers would improve with better sensor calibration and finely tuned filters.

Table 5: Performance of UKF IMU/GNSS/DVL integration schemes for different attitude noise parameterization: rotation vector vs. quaternion vector part (LC - loosely-coupled, TC - tightlycoupled).

\begin{tabular}{|c|c|c|c|c|}
\hline & \multicolumn{2}{|c|}{$\begin{array}{c}\text { Quaternion Unscented KF (UKF) } \\
\text { with Rotation Vector Attitude Noise } \\
\text { Parameterization }\end{array}$} & \multicolumn{2}{|c|}{$\begin{array}{l}\text { Quaternion Unscented KF (UKF) } \\
\text { with Quaternion Vector Part } \\
\text { Attitude Noise Parameterization }\end{array}$} \\
\hline & HPE, [m] & VPE, [m] & HPE, [m] & VPE, [m] \\
\hline LC IMU/GNSS/DVL: FOG + RTK & 19.55 & 5.26 & 19.32 & 5.49 \\
\hline LC IMU/GNSS/DVL: FOG + SPP & 16.53 & 7.19 & 16.30 & 7.45 \\
\hline TC IMU/GNSS/DVL: FOG & 17.27 & 7.34 & 16.43 & 6.16 \\
\hline LC IMU/GNSS/DVL: MEMS + RTK & 17.26 & 8.03 & 17.29 & 8.18 \\
\hline LC IMU/GNSS/DVL: MEMS + SPP & 14.71 & 9.84 & 14.72 & 10.02 \\
\hline TC IMU/GNSS/DVL: MEMS & 17.72 & 9.92 & 14.72 & 10.12 \\
\hline
\end{tabular}

Figure 3 confirms that for all filter configurations and complete 3D DVL measurement model the position drift (both in terms of HPE and VPE) becomes close to linear in time, while pure inertial mechanization shows cubit time dependence (see Figure 4.). This can be easily explained by the fact, that within the INS mechanization (chain of several integrators) the DVL observation (velocity) is placed closer to the position output compared to the inertial measurements. Therefore, in DVL-augmented system the quality of the IMU plays a dominating role only in determining the associated attitude of the system, but because even MEMS IMU has a bias stability of 6 deg/hour, longer GNSS outages could be necessary in order see the impact of attitude accuracy on the estimated position. Here the combined IMU/GNSS/DVL system reduces requirements to the quality of the inertial sensors which is an important step for wider adoption of the proposed navigation strategy. Although there seems to be fairly minor difference between filter configurations (looselyvs. tightly-) if the DVL measurements are available on a regular basis, one could still prefer 
to work with tightly-coupled KFs due to other advantages such as ability to work with direct observations, navigation with less than four satellites etc.

One should also notice a failure of 2D DVL model in terms of VPE as shown in Figure 4 (right). This interesting behavior along an unobserved direction, probably, again comes from DVL modeling errors. This is an unfortunate result bearing in mind that far better performance is demonstrated using just a pure inertial integration. The results in Table 2 confirm that fully augmented systems need between 2 and 2.5 minutes to accumulate 10 meter errors and can bridge 5 minutes GNSS outages without reaching HPE of 25 meters.

Similar performance is obtained when comparing UKF to alternative SPKF schemes as shown in Tables 3 and 4. Both UKF and CKF perform rather similarly, while SUKF and CDKF fail for the most demanding tightly-coupled approaches. As the mathematical models for the process dynamics are identical in all the filters, the only reason for SUKF and CDKF failure is a higher dimensionality of the tightly-coupled configurations as the original system state is augmented with both pseudorange and Doppler shift measurements. Therefore the augmented state could easily reach $n_{a}=50$, which could be too demanding, especially when one considers numerical issues associated with many orders of magnitude differences in numerical values of the estimated variables and nonlinearities including those due to lever arm compensation and DVL measurements. Although the SUKF and CDKF fail in their basic configuration (we use scaled SUKF), the filters could probably perform fine under additive (non-augmented) noise approach. Still, the obtained results indicate that by stressing the filtering schemes to the limit one can reveal the differences in filter performance which are often not observed in simulated nonlinear "toy" problems. Although the square-root implementation of both CDKF and SUKF could solve some of the issues, one should probably avoid using these two SPKF schemes in highly-dimensional problems such as that presented above. Moreover, the square-root formulations as suggested in [23] for UKF and CDKF, seem to be only quasi square-root filters as the Cholesky update functions could fail for the filter configurations with negative weights [24]. A true square-root CKF implementation had been presented in [24] and seems to be promising candidate for the filters like those discussed in this work both with relatively high dimensionality and measurements of strongly varying quality.

Finally, Table 5 confirms that in practice any of the two attitude noise representations can be used as long as the implementations are consistent and special care is taken to ensure 
that vector part approach does not fail due too large initial convergence as the approach implies the noise to be not larger than the quaternion norm $q^{T} q=1$.

Differently from numerous other authors, we have evaluated the algorithm performance using only real measurement data. As the quality of the estimation is often affected by both the nonlinearity and the mismatched models, the presented approach allows us to address both these issues and provides results which are far more representative for real world applications. Although it is not easy to decouple the influence of both these effects, the modeling and sensor errors seem to play far larger role in limiting the performance of the presented system as so-called Iterated UKF (IUKF) [21] did not show any improvement in HPE figures. What is even more interesting, the IUKF was sometimes performing even worse compared to non-iterative scheme. This could be, probably, explained both by the fact that IMU/GNSS/DVL fusion does not possess any severe nonlinearity and by presence of the modeling errors in the measurement (e.g. DVL's Z-axis pseudomeasurement and GNSS compass geometry errors). Further improvement is expected if special maneuvers are applied in order to improve the observability of some instrument errors and to speed up the filter convergence. Although the preliminary results are promising, the system performance is strongly dependent on observability of some sensor errors and is conditioned by the dynamics of the vessel exactly before and during the GNSS outage. Here the richness of the associated dynamics could have an extreme influence on the final performance of this multi-sensor system. The presented approach is consistent with the development of the e-Navigation strategy and results in an affordable setup due to lower costs with a promising potential for both performance and robustness improvement due to constantly increasing quality of inertial MEMS sensors.

\section{Summary and Outlook}

This work had presented an integrated navigation algorithm for maritime applications using SPKF-based nonlinear filtering framework. The proposed algorithms solve the multi-sensor fusion problem for a hybrid navigation system using inertial, GNSS and DVL measurements. While employing real sensor measurements recorded during typical vessel operations we were able to demonstrate the proposed system successfully bridging the GNSS outages of prolonged duration. The results clearly indicate that the addition of DVL to classical IMU/GNSS solution significantly reduces the position drift when GNSS data is not available and the performance of the methods is consistent for both loosely- and tightly-coupled systems with inertial sensors of different accuracy classes. CKF-based approaches seem to be the best option due to its robust performance (similarly to that 
shown by UKF), but with existing efficient true square-root implementation compared to quasi square-root version of UKF. Still, similarity of UKF and CKF performance should be not surprising as CKF can be shown to be particular case of UKF with $\alpha=1, \beta=0$ and $\kappa=0$.

Future work will focus on extending the proposed hybrid system for GNSS phase measurements and implementation of the associated integrity monitoring algorithms. Some further research is also planned in improving the sensor models with proper treatment of correlated noises, sensor misalignments and scale factor errors as well as incorporation of GBAS correction data. Special attention should be paid to the performance of the DVL both in deeper water (when measuring speed through water) and during the berthing situation when the wake under the keel could result in reduced performance of the sensor.

\section{Acknowledgement}

The authors would like to thank Mr. Carsten Becker, Mr. Uwe Netzband and Dr. Stefan Gewies for their support in measurement campaigns as well as for building and maintaining the PNT hardware and software.

\section{References}

[1] H. C. H. G. of Experts on Safety of Navigation, "Report on shipping accidents in the baltic sea during 2011, Std. HELCOM SAFE NAV3/2013," Malmo, Tech. Rep., 5 February 2013.

[2] R. Ziebold, Z. Dai, T. Noack, and E. Engler, "Concept for an integrated pnt-unit for maritime applications," in Satellite Navigation Technologies and European Workshop on GNSS Signals and Signal Processing (NAVITEC), 2010 5th ESA Workshop on, December 2010, pp. 1-8.

[3] T. Moore, C. Hill, A. Norris, C. Hide, D. Park, and N. Ward, "The potential impact of GNSS/INS integration on maritime navigation," The Journal of Navigation, vol. 61, p. 221237, 2008.

[4] E. Kraft, "A quaternion-based unscented Kalman filter for orientation tracking," in Information Fusion, 2003. Proceedings of the Sixth International Conference of, vol. 1, July, pp. 47-54. 
[5] R. Ziebold, M. Romanovas, and L. Lanca, Activities in Navigation. Marine Navigation and Safety of Sea Transportation. CRC Press, 2015, ch. Evaluation of Low Cost Tactical Grade MEMS IMU for Maritime Navigation, pp. 237-246.

[6] Y. C. Lee and D. G. O'Laughlin, "A performance analysis of a tightly coupled GPS/inertial system for two integrity monitoring method," Navigation, vol. 47, no. 3, pp. 175-189, 2000.

[7] N. El-Sheimy, E.-H. Shin, and X. Niu, "Kalman filter face-off: Extended vs. unscented Kalman filters for integrated GPS and MEMS inertial," Inside GNSS, March 2006.

[8] J. Wendel, A. Maier, J. Metzger, and G. F. Trommer, "Comparison of extended and sigma-point Kalman filters for tightly coupled GPS/INS integration," in AIAA Guidance, Navigation and Control Conference and Exhibit, San Francisco, California, 15-18 August 2005.

[9] T. Ford, J. Hamilton, M. Bobye, and L. Day, "GPS/MEMS inertial integration methodology and results," NovaTel, Tech. Rep., 2004.

[10] J. McMillan, "Mins-b ii: a marine integrated navigation system," in Position Location and Navigation Symposium, 1988. Record. Navigation into the 21st Century. IEEE PLANS '88., IEEE, Nov 1988, pp. 499-508.

[11] L. L. W. James C Kinsey, Ryan M Eustice, "A survey of underwater vehicle navigation: Recent advances and new challenges," in IFAC Conference of Manoeuvering and Control of Marine Craft, 2006.

[12] L. Stutters, H. Liu, C. Tiltman, and D. Brown, "Navigation technologies for autonomous underwater vehicles," Systems, Man, and Cybernetics, Part C: Applications and Reviews, IEEE Transactions on, vol. 38, no. 4, pp. 581-589, July 2008.

[13] M. B. Larsen, "High performance autonomous underwater navigation," Hydro International, vol. 6, pp. 2043-2050, 2002.

[14] B. Jalving, K. Gade, K. Svartveit, A. Willumsen, and R. Srhagen, "DVL Velocity Aiding in the HUGIN 1000 Integrated Inertial Navigation System," Modeling, Identification and Control, vol. 25, no. 4, pp. 223-236, 2004.

[15] S. Thrun, W. Burgard, and D. Fox, Probabilistic Robotics (Intelligent Robotics and Autonomous Agents). The MIT Press, September 2005. 
[16] S. Julier and J. Uhlmann, "A new extension of the Kalman filter to nonlinear systems," in Int. Symp. Aerospace/Defense Sensing, Simul. and Controls, Orlando, FL, 1997.

[17] S. Julier, "The spherical simplex unscented transformation," in American Control Conference, 2003. Proceedings of the 2003, vol. 3, june 2003, pp. $2430-2434$ vol.3.

[18] S. Roumeliotis, G. Sukhatme, and G. A. Bekey, "Circumventing dynamic modeling: evaluation of the error-state Kalman filter applied to mobile robot localization," in Robotics and Automation, 1999. Proceedings. 1999 IEEE International Conference on, vol. 2, 1999, pp. 1656-1663 vol.2.

[19] K. Borre and G. Strang, Algorithms for Global Positioning. Wellesley-Cambridge Press, 2010.

[20] T. Takasu and A. Yasuda, "Development of the low-cost RTK-GPS receiver with an open source program package RTKLIB," in International Symposium on GPS/GNSS, 2009.

[21] R. Zhan and J. Wan, "Iterated unscented Kalman filter for passive target tracking," IEEE Transactions on Aerospace and Electronic Systems, vol. 43, no. 3, pp. 11551162, 2007.

[22] W. Khoder, B. Fassinut-Mombot, and M. Benjelloun, "Quaternion unscented Kalman filtering for integrated inertial navigation and GPS," in Information Fusion, 2008 11th International Conference on, June 2008, pp. 1-8.

[23] Rudolph van der Merwe. „Sigma-point kalman filters for probabilistic inference in dynamic state-space models," Workshop on Advances in Machine Learning, Montreal, June 2003.

[24] Arasaratnam and S. Haykin. "Cubature Kalman filters," IEEE Transactions on Automatic Control, ,54 (6):1254-1269, 2009. 